\title{
OPTICAL PROBES OF INTRADISKAL PROCESSES IN ROD PHOTORECEPTORS \\ I: LIGHT-SCATTERING STUDY OF ATP-DEPENDENT DARK REACTIONS
}

\section{RAINER UHL ${ }^{\dagger}$, RENATE ZELLMANN-KRASKA and HERBERT DESEL}

Max-Planck-Institut für biophysikalische Chemie, Am Fassberg, D-3400 Göttingen (F.R.G.)

(Received August 5, 1988; accepted November 16, 1988)

Keywords. Photoreceptor disk compartment, light-scattering changes in the dark, Mg-ATPase, proton translocation, $\Delta \mu\left(\mathrm{H}^{+}\right)$.

\section{Abbreviations}

$\begin{array}{ll}\text { ATP } & \text { adenosine } 5^{\prime} \text {-triphosphate } \\ \Delta \mu\left(\mathrm{H}^{+}\right) & \text {electrochemical potential difference of protons } \\ \text { BICINE } & N, N \text {-bis(2-hydroxyethyl)glycine } \\ \text { CAPS } & 3 \text {-(cyclohexylamino)-1-propanesulphonic acid } \\ \text { cGMP } & \text { guanosine } 3^{\prime}: 5^{\prime} \text {-cyclic monophosphate } \\ \text { DCCD } & N, N^{\prime} \text {-dicyclohexylcarbodiimide } \\ \text { DIDS } & 4,4 \text {-diisothiocyanatostilbene-2,2'-disulphonic acid } \\ \text { EGTA } & \text { ethylene glycol-bis }\left(b \text {-aminoethylether)- } N^{\prime}, N^{\prime}, N^{\prime}, N^{\prime} \text {-tetraacetic }\right. \\ & \text { acid } \\ \text { HEPES } & \left(N \text {-[2-hydroxyethyl]-piperazine- } N^{\prime} \text {-[2-ethanesulphonic acid]) }\right. \\ \text { MES } & \begin{array}{l}2 \text {-( } N \text {-morpholino)-ethanesulphonic acid } \\ \text { NBD·Cl }\end{array} \\ \text { 7-chloro-4-nitrobenz-2-oxa-1,3-diazole } \\ \text { PEP } & \text { phosphoenolpyruvate } \\ \text { PIPES } & 1,4 \text {-piperazinediethanesulphonic acid } \\ \text { ROS } & \text { rod outer segment }\end{array}$

\section{Summary}

ATP can cause dramatic structural changes in the outer segment of rod photoreceptors. These changes can be visualized by means of a concomitant light-scattering signal $A_{D}$, a decrease in scattered light intensity of over $20 \%$. The large size of the signal suggests that major structural changes occur. The underlying molecular events may reflect an important, yet still unknown, part of the photoreceptor machinery.

†Author to whom correspondence should be addressed. 
$A_{D}$ signals reflect ATPase-driven transmembrane events which occur in and at the disk membrane. Their only structural prerequisite is the structural integrity of the disk compartment. The angular dependence of $A_{D}$, which can be mimicked by an osmotically-induced disk-swelling, suggests that the disk compartment swells during the production of the $A_{D}$ signal.

$A_{D}$ signals proceed with first-order kinetics (half-life $=1 \mathrm{~min}$ at $20^{\circ} \mathrm{C}$ and ATP concentrations of greater than $100 \mu \mathrm{M}$ ) and are accompanied by the hydrolysis of approximately $4 \mathrm{~mol}$ ATP (mol rhodopsin) $)^{-1}$.

The $A_{D}$ signal is inhibited by a number of transport ATPase inhibitors (quercetin, $\mathrm{NBD} \cdot \mathrm{Cl}$, vanadate, DCCD), but not by oligomycin, azide and ouabain. The sensitivity to DCCD, together with the fact that except magnesium no other cation has to be present, points to a proton translocation. This proton transport appears to be electrogenic, since $A_{D}$ signals require the presence of a permeant anion. In physiological saline this is chloride, and the chloride flux is facilitated by a DIDS-sensitive anion transport unit in the disk membrane.

\section{Introduction}

Our understanding of the mechanism of visual transduction has progressed considerably over the past few years. A sequence of biochemical events has been discovered, which starts in the disk membrane with the absorption of a photon by the pigment molecule rhodopsin, proceeds at the disk membrane surface with the transient activation of many G-proteins and, subsequently, phosphodiesterase molecules, and terminates with the blockage of cGMP-dependent sodium channels in the plasma membrane of the rod photoreceptor [1]. Within the framework of this model the disk membrane merely serves as a two-dimensional reaction vessel, facilitating the steric interaction between the various proteins. Its flatness affords a favourable orientation of the chromophore and the fact that rhodopsin is transmembrane provides for a solid anchoring. If this is so, why do disks form topologically closed membrane compartments (like thylakoids or mitochondria) and why does rhodopsin cause several light-induced intradiskal events? Moreover, what is the reason for the curious fact that the intradiskal loops of the rhodopsin molecule are among the best conserved sequences in vertebrate and drosophila rhodopsin [2]? These unanswered questions have suggested to us that the disk compartment may possibly play a role in visual transduction, a role which has yet to be determined.

A first step towards a substantiation of this idea has been undertaken in this and a following paper, where we have tried to identify further properties of the disk compartment and to measure dark and light reactions which take place inside the disk, rather than at its cytosolic surface. The physiological significance of our findings is, at present, unclear. However, 
we believe that it is important to continue to gather information in order to try to produce a clearer picture of what is taking place.

Optical techniques can be used to study processes which occur in an unperturbed disk interior. Of these techniques, light scattering is probably the most sensitive. However, since it rarely leads to clear results a more logical approach has to be taken: assumptions must be made, further assumptions must be made which lead to further experiments (possibly justifying the previous assumptions) and a large amount of experimental evidence must be collected before a reasonably clear picture is obtained. The purpose of this and a following paper is to present large amounts of experimental data on the photoreceptor disk compartment and to demonstrate strategies for the extraction of information from these data.

Since our first systematic studies of time-resolved, light-induced, lightscattering transients from bovine ROS material [3 - 6], light scattering has been used to aid in the understanding of light-stimulated protein-protein and protein-membrane interactions, which all occur at the cytosolic disk surface. The basis for this progress has been the observation of Kühn et al. [7] that certain processes, involved in the light activation of rod photoreceptor enzymes, are accompanied by gross structural changes, and that these structural changes are reflected by the well-known light-scattering signais.

In the late 1970s a new class of light-scattering signals from ROS was discovered [8-13]. The signals are ATP dependent and, as is demonstrated in this and a following paper, they reflect trans-disk membrane processes rather than processes involving peripheral disk membrane proteins. These signals are a large decrease in light scattering (greater than 20\%) in the dark (termed $A_{\mathrm{D}}$ ) and a subsequent further decrease in light scattering (termed $A_{\mathrm{L}}$ ) which is observed when ROS pre-incubated with ATP is flash illuminated.

In this paper we call the process which produces the $A_{D}$ signal (and is a prerequisite for $A_{L}$ ) energization of the disk, simply because it builds up at the expense of metabolic energy, e.g. ATP. Evidence is provided later in this and the following paper that the ATP-driven energization of the disk actually reflects the development of an electrochemical proton potential difference $\Delta \mu\left(\mathrm{H}^{+}\right)$as in mitochondria and thylakoids.

\section{Materials and methods}

\subsection{Chemicals}

Ionophores and inhibitors were stored as concentrated ethanolic stock solutions in the dark at $4{ }^{\circ} \mathrm{C}$. They were mixed with the test suspension using a microsyringe. The ethanol concentration in the reaction medium never exceeded $0.5 \%$. X537A and nigericin were provided by Dr. W. E. Scott, Hoffmann LaRoche. A23187 was obtained from Calbiochem, tributyltin from Aldridge, Ficoll 400 from Pharmacia and all other reagents from Sigma. 


\subsection{Preparation of rod outer segment (ROS) material}

\subsubsection{Bovine ROS}

Bovine ROS fragments with intact stacks of disks but perforated plasma membranes were prepared using a procedure described in ref. 14 . Briefly, ROS fragments were released from fresh retinae by vortexing for $5 \mathrm{~s}$ in argonated Ringer's solution. Crude ROS fragments were separated from retinal debris by filtering them through a Nylon mesh. They were purified further using discontinuous sucrose density gradient centrifugation. The usual yield was approximately $20 \mathrm{nmol}$ rhodopsin per retina and the absorbance ratio $A_{280} / A_{500}$ (indication of purity) was between 2.2 and 2.4. The ROS fragments were stored as small aliquots in liquid nitrogen and were thawed immediately before a particular experiment.

\subsubsection{Disks}

Isolated, freely floating disks were prepared from unfrozen ROS material using the Ficoll flotation procedure of Smith et al. [15]. They were harvested as swollen vesicles from the Ficoll-phosphate buffer interface (the overlayering with $100 \mathrm{mM}$ sodium phosphate $(\mathrm{pH} 7.2)$ is a modification of the original Smith procedure), and were used immediately afterwards. It is important to note that $A_{D}$ signals can only be obtained from disk preparations that have not been frozen. This seems to indicate that disks are stabilized by the stack and cannot withstand freezing when they are isolated.

Frequently in our experiments isolated disks were replaced by lysed ROS material. This was prepared by incubating the ROS for $20 \mathrm{~min}$ in $5 \mathrm{mM}$ HEPES ( $\mathrm{pH} 7.2$ ) and $1 \mathrm{mM} \mathrm{MgSO}_{4}$. Subsequently the material was pelleted in small Eppendorf vials, using an Eppendorf centrifuge, and resuspended in the particular measuring medium [14].

Most experiments reported in this paper were carried out using freezethaw-treated ROS. The experiments were reproduced using fresh material which was lysed immediately prior to the experiment.

\subsubsection{Frog ROS}

Bullfrogs (Rana catesbiana) were adapted to the dark over night and decapitated. The isolated retinae were shaken vigorously in Ringer's solution identical with that used for bovine retinae, except that the sodium concentration was only $90 \mathrm{mM}$. This procedure, which was performed within $5 \mathrm{~min}$ of the death of the animal, yielded a crude ROS suspension with mostly intact plasma membrane [16]. It was used without further purification in a number of experiments.

\subsection{Light-scattering experiments}

Light-scattering signals were recorded in a multiangle flash photolysis apparatus (MAFPA), the design of which has been described elsewhere [13]. It allows the simultaneous registration of light-scattering signals at eight scattering angles, has a time resolution of $32 \mu$ s and can resolve relative changes in light intensity as small as $0.004 \%$. 
The signals displayed in this paper usually reflect the decrease in scattered light intensity at an angle of $8^{\circ}$, where $A_{D}$ signals have their highest amplitude. In Fig. 3 (see later) previous measurements are shown which were recorded at a fixed scattering angle of $10^{\circ}$. In Figs. 5, 6 and 11 (see later) the amplitudes of $A_{D}$ were determined from the drop in transmission measured in a Zeiss PMQ-2 photometer. In all cases the wavelength of the monitoring light was $840 \mathrm{~nm}$.

To avoid multiple scattering, a rhodopsin concentration of $1 \mu \mathrm{M}$ was chosen. In a cuvette with a path length of $5 \mathrm{~mm}$ this corresponds to a transmission of approximately $80 \%$.

Unless otherwise indicated in the figure captions, the measuring buffer contained $100 \mathrm{mM} \mathrm{KCl}, 5 \mathrm{mM}$ PIPES (pH 7.2) and $1 \mathrm{mM} \mathrm{MgCl}_{2}$. The usual Mg-ATP concentration was $100 \mu \mathrm{M}$ and the temperature was $22{ }^{\circ} \mathrm{C}$.

\subsection{ATPase activity measurement}

The A'T'Pase activity was determined using an ATP-regenerating system (phosphoenolpyruvate-pyruvate kinase) coupled to the NADH-dependent reduction of pyruvate to lactate [17]. The rate of the absorbance decrease at $340 \mathrm{~nm}$, corrected for light-scattering effects, was used as a measure of ATPase activity [11]. It was either determined in an Aminco DW-2 dual wavelength spectrophotometer (wavelength pair: 340 and $400 \mathrm{~nm}$ ) or, in previous experiments (Fig. 3, see later), in a Zeiss PMQ-2 with manual lightscattering correction, using the same set of wavelengths.

\section{Results}

When ROS fragments with a perforated plasma membrane are incubated in the presence of Mg-ATP, their light-scattering behaviour changes dramatically with 5 min [10]. Figure 1 shows such an $A_{D}$ signal, monitored as a decrease in turbidity at a scattering angle of $0^{\circ}$ and as a decrease in scattered light intensity at a scattering angle of $8^{\circ}$. Since nonhydrolysable ATP analogues cannot substitute for ATP, and even suppress $A_{D}$ when added together with ATP, we conclude that the underlying structural phenomenon requires the hydrolysis of ATP [9]. Neither GTP nor creatine phosphate produce a similar effect.

\subsection{A D $_{\mathrm{D}}$ signals are physiological}

$A_{D}$-type signals can also be observed in freshly prepared (frog) ROS with completely intact plasma membrane (data not shown). Here, because of the presence of endogenous ATP (we verified this for our preparation using a luciferase-luciferin assay; data to be published), the addition of exogenous ATP is no longer required. Instead, the ROS must be kept on ice for a while and then warmed up in order to initiate $A_{D}$. The ATP-dependent energization of the disk is apparently unstable at low temperature (because the ATPase activity is sufficiently slowed down) and so the energization can take place again on warming. After perforation of the plasma membrane 

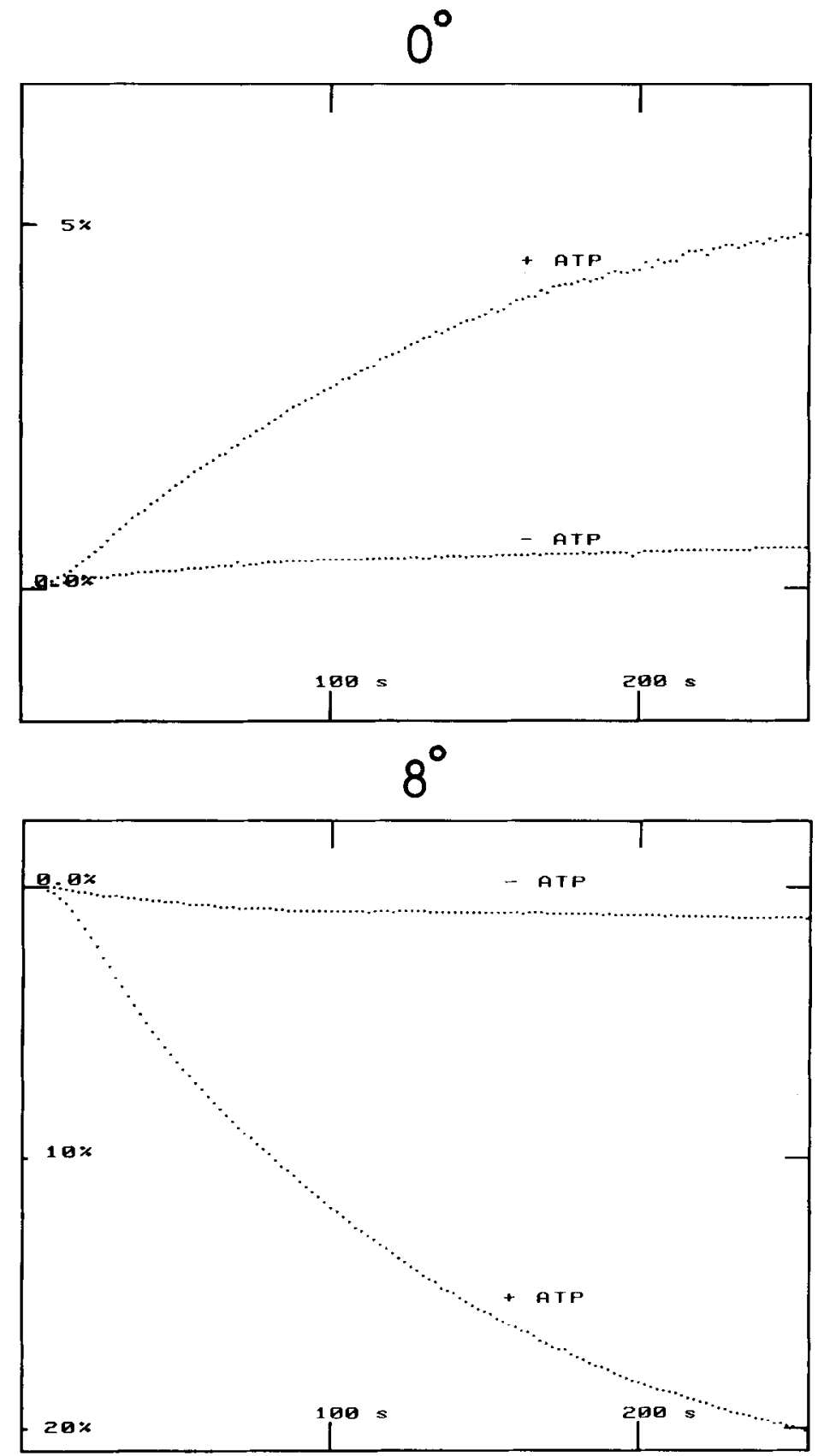

Fig. 1. A signals at $0^{\circ}$ (increase in transmission) and at a scattering angle of $8^{\circ}$ (decrease in scattered light intensity). The rhodopsin concentration was $1 \mu \mathrm{M} M g \cdot A T P(100 \mu \mathrm{M})$ was added immediately prior to measurement. The signal obtained at $0^{\circ}$ is small because the turbidity of the suspension was very low to avoid multiple scattering in the cuvette. Charges of up to $40 \%$ can be obtained when the rhodopsin concentration is increased tenfold. 
by a freeze-thaw cycle, the frog ROS material still produces an $A_{D}$-type signal on resuspension in warm measuring buffer. This signal, like $A_{D}$, can be inhibited by vanadate. It is also blocked by a large excess of glucose and hexokinase. It follows that even broken ROS material retains sufficient amounts of ATP to drive $A_{D}$ signals to completion [16].

\subsection{ATPase activity during $A_{D}$}

Figure 2 shows that ROS material exhibits ATPase activity which runs in parallel with the $A_{D}$ signal. It can be monitored as an initial burst of ATPase activity on top of a constant time-independent activity. The initial activity corresponds to the hydrolysis of about 4 mol ATP (mol rhodopsin $)^{-1}$. Its inhibitor specificity is identical with that of $A_{D}$ (Fig. 3), suggesting that there is a link between the two processes.

The second persistent phase of ATPase activity, however, which is completely blocked by quercetin, is only slightly sensitive to vanadate and DCCD and is therefore distinct. We assume that its major, quercetinsensitive, vanadate-insensitive part reflects the Mg-ATPase activity described by Thacher [18], whereas its small vanadate-sensitive fraction reflects the ATP consumption required for the maintenance of the energized state. The amplitude of the latter was determined in a number of experiments (data not shown) to be $0.2-0.3 \mathrm{~mol}$ ATP hydrolysed $\mathrm{min}^{-1}$ (mol rhodopsin) $)^{-1}$. This corresponds to a decay rate of the previous energization of $7 \% \mathrm{~min}^{-1}$ or a half-life of de-energization of $10 \mathrm{~min}\left(\right.$ at $22^{\circ} \mathrm{C}$ ).

The fact that $A_{D}$ is inhibited by the transport ATPase inhibitors DCCD and quercetin (Fig. 3) and also by $\mathrm{NBD} \cdot \mathrm{Cl}$ (data not shown) points to a transport ATPase; the DCCD sensitivity suggests that proton translocation is involved and the insensitivity to azide precludes the possible participation of an $F_{1} / F_{0}$-type enzyme. This, together with the huge size of the lightscattering signal, clearly establishes that the underlying events originate in the ROS and not in contaminating (mitochondrial) material.

\subsection{Kinetic characterization of $A_{D}$}

Micromolar amounts of ATP are sufficient for $A_{D}$ signals to occur [10]. Their rate increases with increasing ATP concentration in a manner predicted by the Michaelis Menten equation when a 1:1 stoichiometry between ATPase and ATP is assumed. The $K_{M}$ value was determined to be approximately $60 \mu \mathrm{M}$, and the activation energy is $45 \mathrm{~kJ} \mathrm{~mol}^{-1}$ [16].

\subsection{The structural origin of $A_{D}$}

Thacher [19] has characterized $A_{D}$ signals from frog ROS. He detected no signs of a disk swelling during $A_{D}$, using both light microscopy and radioactive tracer experiments. He therefore concluded that the ATPase in question is not a transport ATPase. He suggested that il may be a dyneintype ATPase similar to those connected with the microtubular structures of eucanyotic flagellae. These ATPases are also sensitive to vanadate, and ciliary structures have been reported to exhibit similarly pronounced light- 

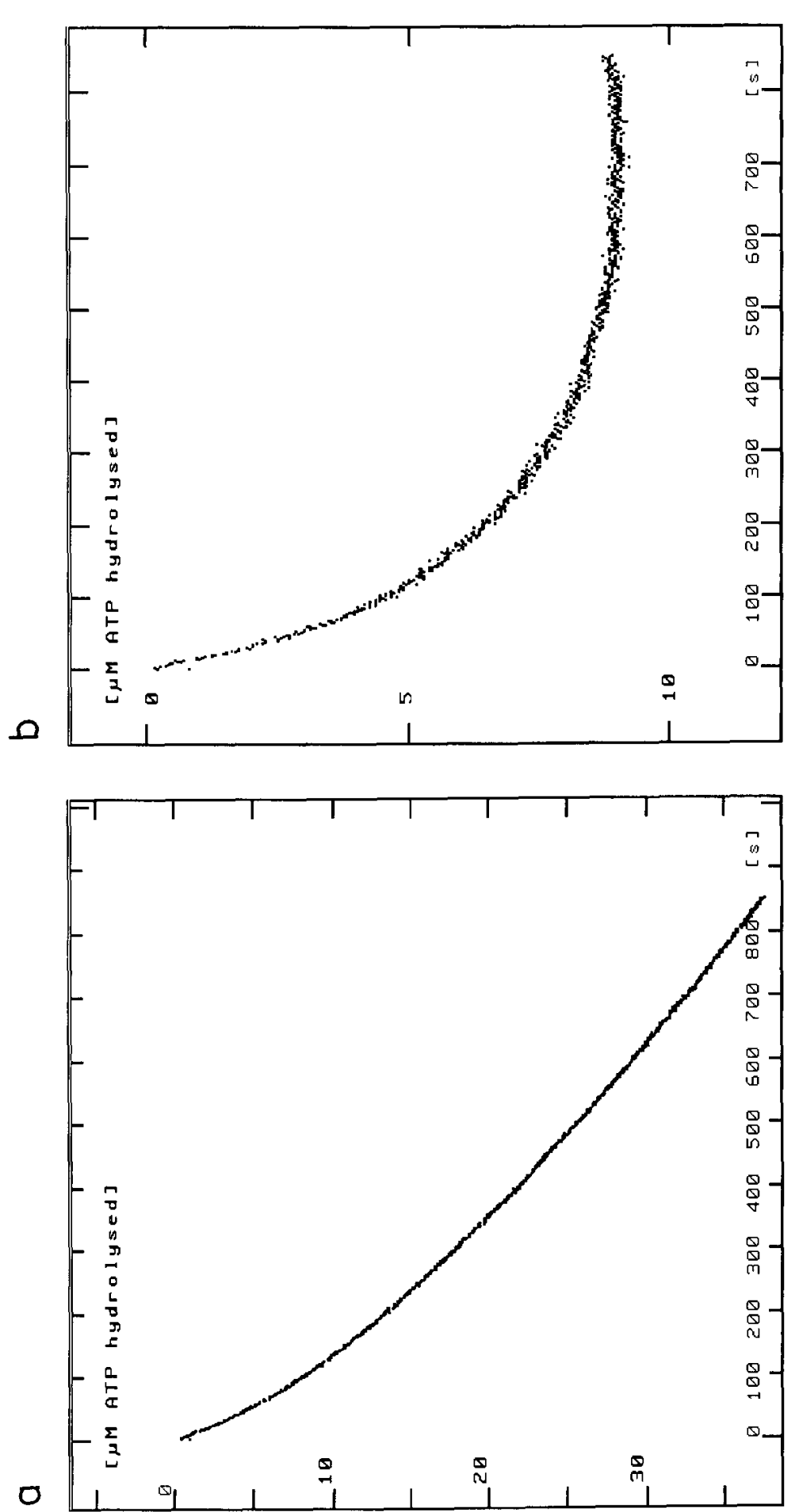

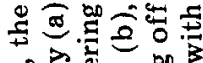

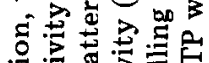

记

完

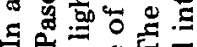

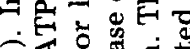

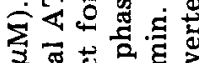

西望西是

ᄂ

द

용

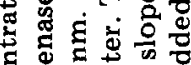

品

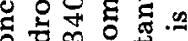

8 范要

뒁

웜

웡 要远

द

๙

金苛.

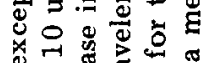

일

퍼웛

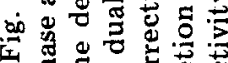

$\Xi \Xi_{N}$ 过

$\Xi$ 的

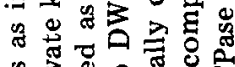

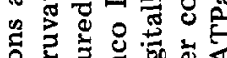

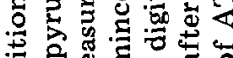

设

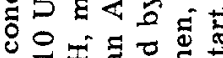

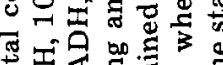

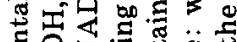

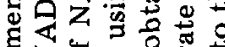

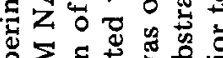

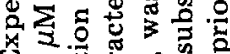

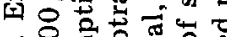

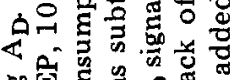

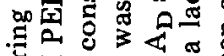

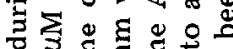

$\rightarrow ㅇ ㅕ ㄴ$

녹윽 동용웜

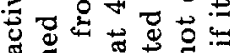

\%

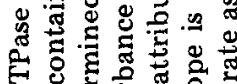

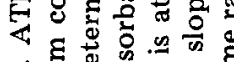

ง

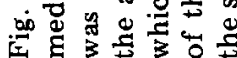




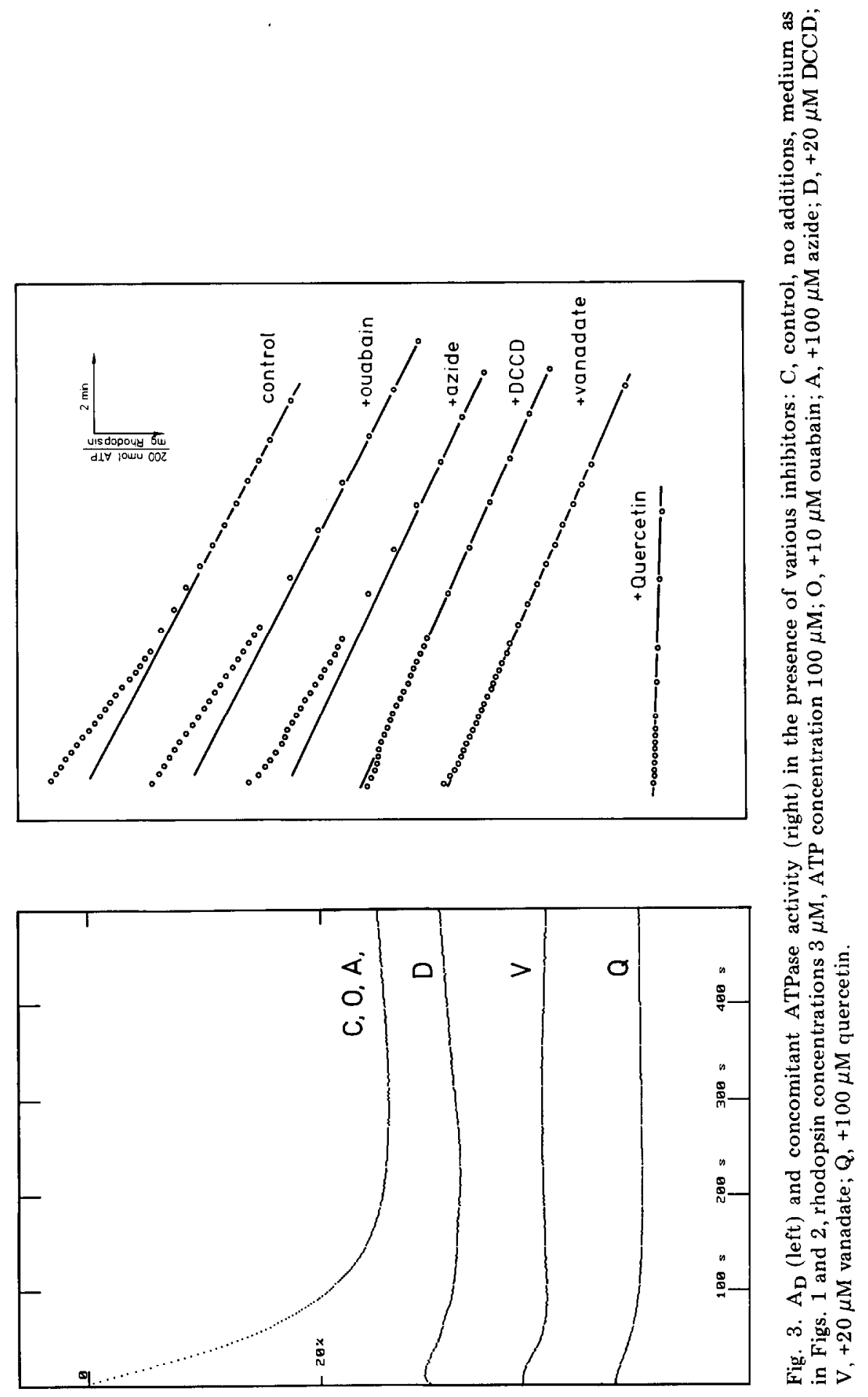


scattering signals in the presence of ATP (see ref. 19). The recently discovered filaments connecting the rim of adjacent disks [20] would be candidates for such structures and could hence be responsible for $A_{D}$. However, the results of this and a following paper do not support this interpretation.

We have also attempted to clarify the structural origin of $A_{D}$ and we have therefore determined its angular dependence (Fig. 4). It is basically the same for stacked disks, lysed ROS (data not shown) and freely floating, isolated disks, thus ruling out the participation of vesicle aggregation effects and demonstrating that neither a disk stack nor peripheral ROS proteins are essential. The only structure which all three preparations have in common (see also the electron micrographs in refs. 14 and 15) is the disk itself, indicating that it must be the disk structure that undergoes the ATPdependent changes.

Interpreting light-scattering data from ROS material by theoretical means is exceedingly difficult [21] and has hardly ever led to meaningful conclusions. Therefore, we tried to mimick the ATP-dependent lightscattering changes during $A_{D}$ by osmotically-induced volume changes. An identical angular dependence is observed when disk swelling is produced by the addition of the potassium ionophore valinomycin $(2 \mu \mathrm{M})$ to disks suspended in a medium containing KSCN (Fig. 4). Thiocyanate is a lipid-soluble anion which promotes net salt uptake, provided that there is a conductance for the particular cation. The similarity of the angular profiles leads us to the conclusion (which will be verified repeatedly later on) that the structural origin of $A_{D}$ is also a swelling of the disk compartment due to the uptake of salt and water.

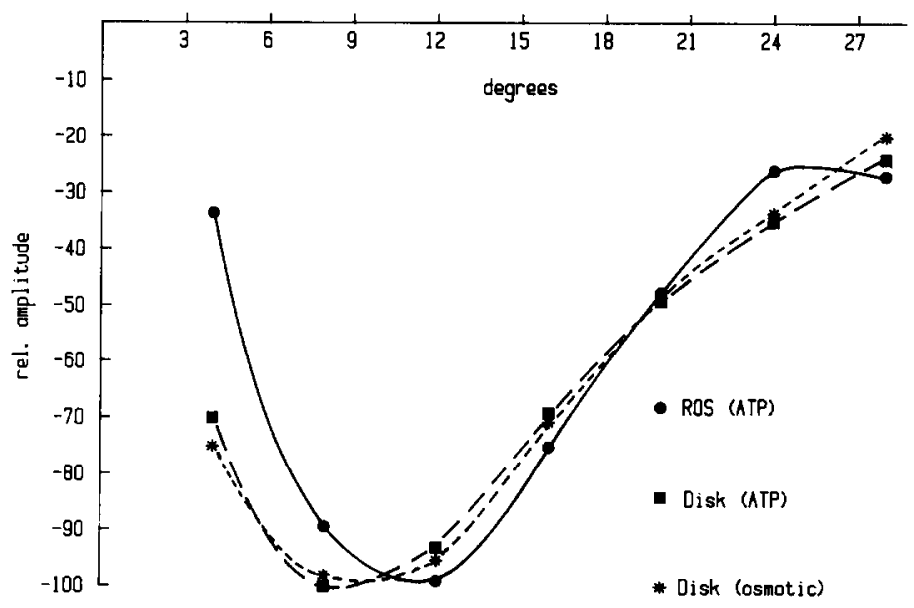

Fig. 4. Angular dependence of $A_{D}$ signals obtained from ROS and from freely floating, isolated disks. For comparison the angular dependence of an osmotically-induced disk swelling is also shown. 


\section{5. $A_{D}$ signal and disk volume}

The disk compartments of ROS fragments, suspended in media of different osmotic strength, behave as (not ideal) osmometers [22]. Figure 5 shows that the amplitude of $A_{D}$ depends strongly on the osmolarity and therefore on the disk volume. The peculiar behaviour in sucrose medium, i.e. the increase in amplitude at very high osmolarities, is consistent with the observation of Berman et al. [23] that ROS disks are swollen in media of high sucrose concentration. There is, so far, no explanation for this effect, which seems to indicate that, under certain conditions, sucrose can enter the disk. It would also provide a plausible explanation for the observation of Thacher [19] that the internal volume of the disk, as determined by tracer experiments (using labelled sugars), shows unrealistically high values and no increase on addition of ATP.

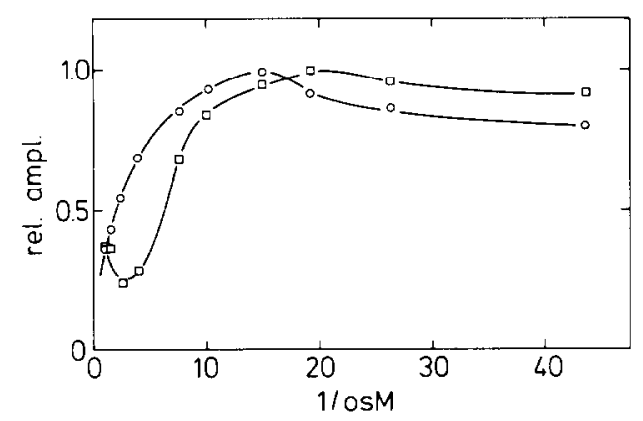

Fig. 5. Amplitude of $A_{D}$ as a function of osmolarity and hence disk volume; $\bigcirc, \mathrm{KCl}$ medium; $\square$, sucrose medium. Experimental conditions as in Fig. 1, except that the respective osmolarities were adjusted using $\mathrm{KCl}$ or sucrose.

Figure 6 shows the dependence of the disk volume, as determined by light scattering, on the osmolarity of the medium. When the ROS fragments are incubated in the presence of ATP prior to the osmotic manipulation, the

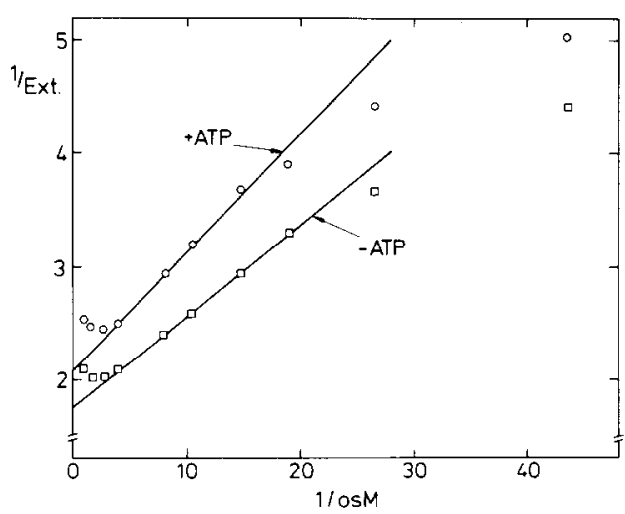

Fig. 6. Turbidity of a ROS suspension, before and after the completion of $A_{D}$ as a function of the osmolarity of the medium. Osmolarities were adjusted with $\mathrm{KCl}$. The reciprocal extinction value is a measure of the disk volume [21]. 
disk volume assumes enhanced values at all tested osmolarities, suggesting that the number of osmotically active ions in the disk interior has increased. However, the extrapolation of the turbidity to infinite osmotic pressure (this should be a measure of the maximally compressed disk volume) also yields an increased value after completion of $A_{D}$. This implies that osmotic effects alone are not sufficient to explain $A_{D}$.

A possible reason for the observed difference in the "compressible volume" of the disks could be an increase in surface potential within the disk, which would impede a closer approach of the two adjacent disk membranes. However, proton transport into the disk interior, as inferred from other experiments, would tend to decrease the surface potential rather than increase it. Moreover, since $A_{D}$ signals are identical in the presence and absence of $50 \mathrm{mM}$ ammonium acetate (data not shown) (a membranepermeable salt that has been reported to destroy any surface potential inside the disk [24]), it must be assumed that surface potentials do not play a major role in the underlying processes of $A_{D}$.

\subsection{Ion specificity of $A_{D}$}

\subsubsection{Divalent and trivalent cations}

The substrate of the ATPase responsible for $A_{D}$ is Mg-ATP. Calcium and barium cannot substitute for magnesium and manganese can only substitute to a small extent (data not shown). Moreover, in the presence of equal amounts of magnesium and calcium $A_{D}$ is inhibited. However, calcium inhibition can be overcome by simply increasing the magnesium concentration. EGTA does not have an inhibitory effect on $A_{D}$; it sometimes stimulates it slightly. Trivalent cations, such as lanthanum, block $A_{D}$ completely, even at very low $(50 \mu \mathrm{M})$ concentration.

\subsubsection{Monovalent cations}

Figure 7 shows that $A_{D}$ signals, recorded in the presence of potassium and choline chloride, are almost identical. The same is true (data not shown) for sodium chloride. However, when the disk membrane becomes permeable to potassium due to the presence of $5 \mu \mathrm{M}$ valinomycin, the signal is markedly enhanced in $\mathrm{KCl}$ and is reduced in choline chloride. This clearly demonstrates that $A_{D}$ is accompanied by transmembrane processes and points to the participation of diffusion potentials. Further support for this comes from the observation that $A_{D}$ can be destroyed by minor amounts of detergents or lysolecithins (data not shown), substances known to cause leaks in membranes.

$\Lambda_{\mathrm{D}}$ can also be observed in the presence of membrane-permeant cations (Fig. 8). The permeable cations used in this study (TRIS, ammonium and imidazolium) are all weak bases. In the presence of a $\mathrm{pH}$ gradient $\Delta \mathrm{pH}$ they redistribute between two aqueous phases according to the proton gradient. A proton uptake by the disk during the course of the $A_{D}$ signal should lead to a concomitant enhanced swelling and hence increase the signal amplitude. The amplitude of the $A_{D}$ signal increases with decreasing 


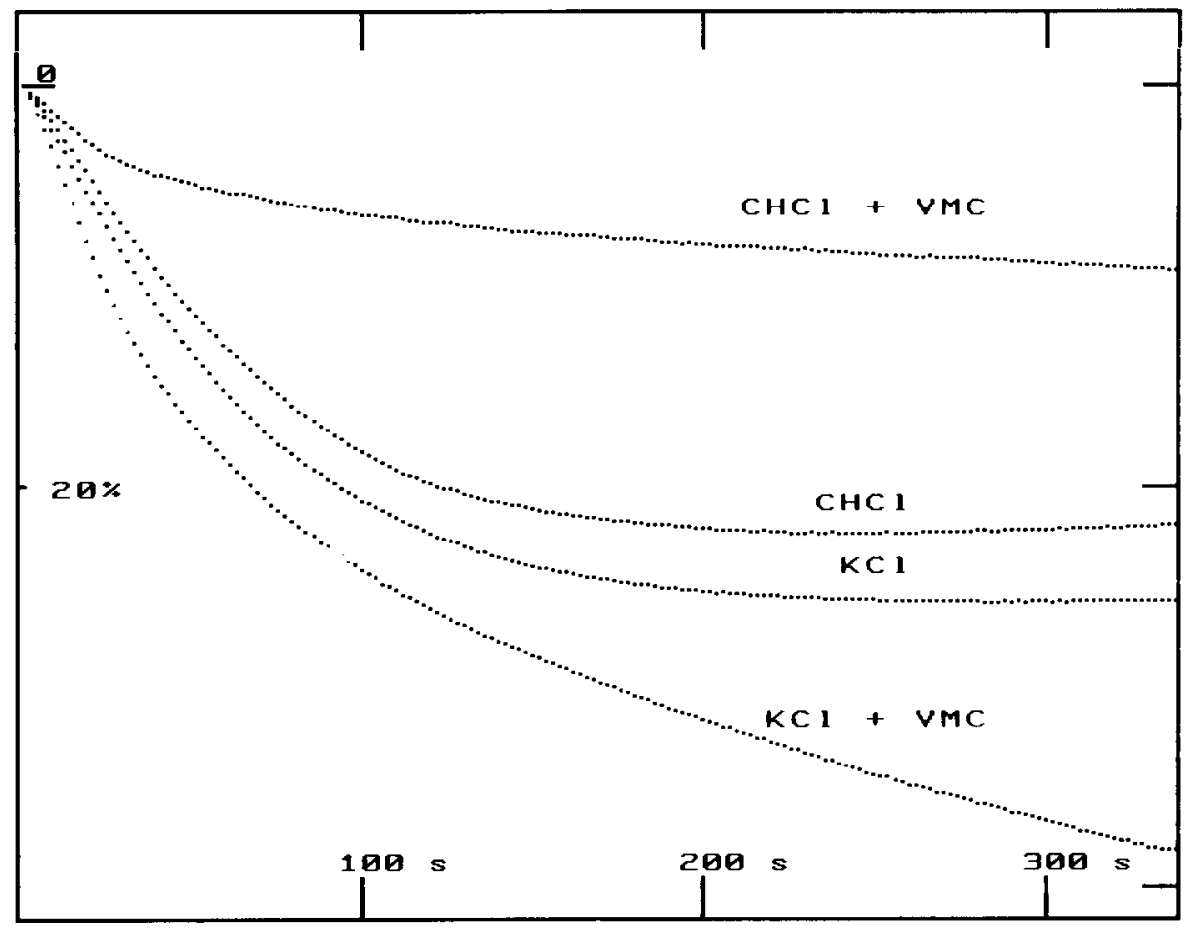

Fig. 7. Effect of $5 \mu \mathrm{M}$ valinomycin on $A_{D}$ signals: ROS fragments were suspended in $100 \mathrm{mM}$ choline chloride $(\mathrm{ChCl})$ or $100 \mathrm{mM}$ potassium chloride $(\mathrm{KCl})$.

$\mathrm{p} K$ value of the permeable cation. Since the fraction of the lipid-soluble, unchanged form also increases with decreasing $\mathrm{pK}$ (at a $\mathrm{pH}$ of $7.2,0.1 \%$ of the ammonia, $10 \%$ of the TRIS and $50 \%$ of the imidazolium molecules are unchanged) this is further support for the assumption of proton uptake by the disk.

Following the same reasoning, $A_{D}$ signals should decrease in size in the presence of lipid-permeable, weak acids. They should leave the disk interior as a consequence of the proposed proton uptake. This has been observed using maleic acid (data not shown).

\subsubsection{Anion specificity}

The anion specificity was tested using the potassium salts of a number of different anions (Fig. 9). The greatest amplitudes are observed in the presence of lipid-soluble anions (thiocyanate, iodide), whereas in the presence of the impermeable, zwitterionic anion PIPES, $A_{D}$ is completely suppressed. The same result is obtained when PIPES is replaced by glutamate (data not shown). From this we can conclude that the light-scattering signal is accompanied by anion uptake. It is interesting to note that $A_{D}$ signals are enhanced, but not accelerated, in the presence of lipophilic anions. This points to the ATPase activity as the rate-limiting factor in the production of the $A_{D}$ signal. 


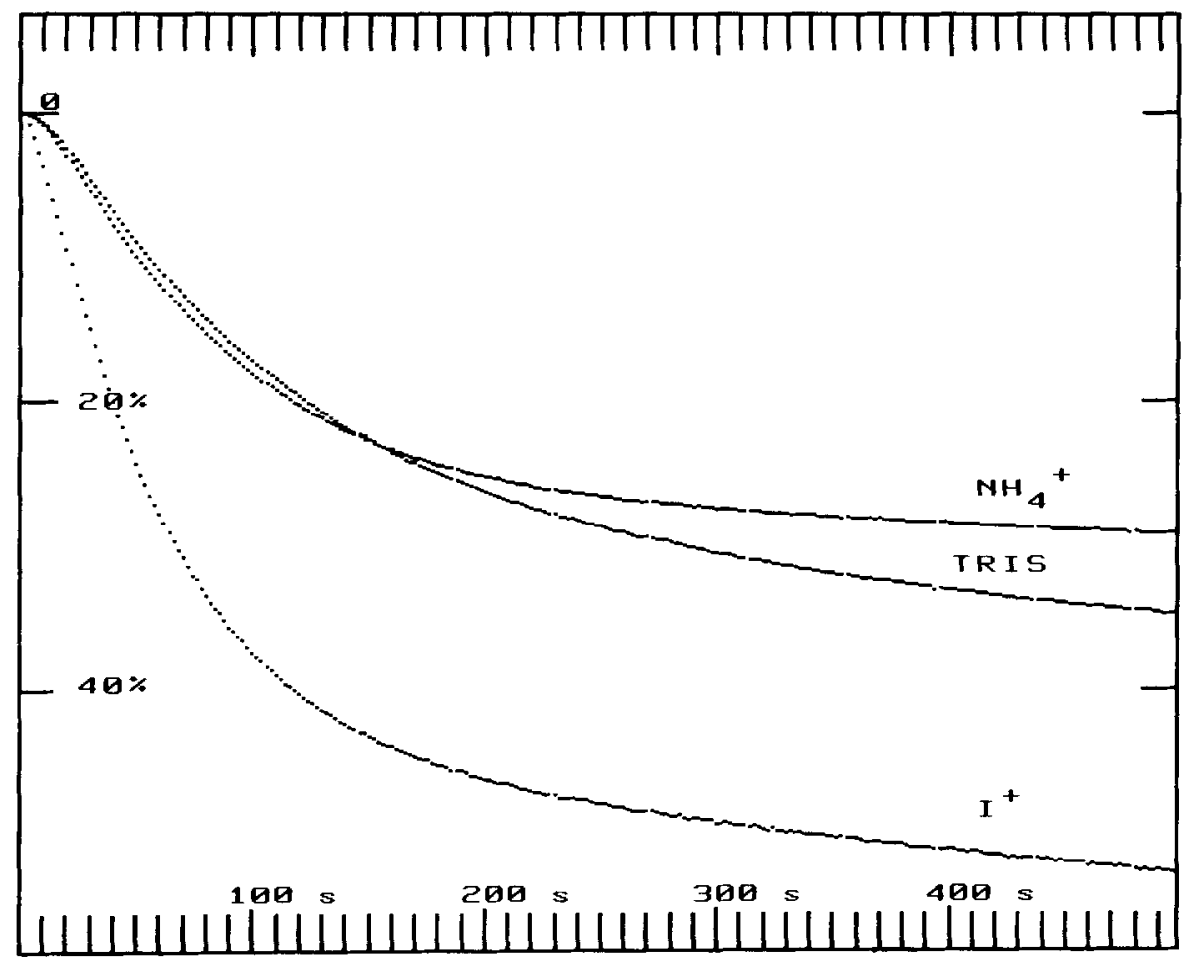

Fig. 8. $A_{D}$ signals obtained in the presence of various permeant cations at $\mathrm{pH} 7.0: \mathrm{I}^{+}$, $100 \mathrm{mM}$ imidazolium chloride; $\mathrm{NH}_{4}^{+}, 90 \mathrm{mM}$ ammonium chloride; TRIS, $100 \mathrm{mM}$ tris$\mathrm{HCl}$.

\subsubsection{Action of the anion channel blocker DIDS}

Further evidence for the participation of anion transport during $A_{D}$ comes from the finding that the anion transport blocker DIDS inhibits $A_{D}$ in the presence of chloride, but not in the presence of the lipophilic thiocyanate anion (Fig. 10).

\subsubsection{Action of ionophores}

In Fig. $10 A_{D}$ signals are shown in the presence and absence of the ionophores tributyltin (a chloride- $\mathrm{OH}^{-}$exchanger) and nigericin (a potassium-proton exchanging antibiotic [22]). The former destroys the signal completely (presumably by reversing the ATP-driven uptake of $\mathrm{HCl}$ ), whereas the latter increases it (it allows the transformation of a proton gradient into an osmotically more effective potassium gradient, hence causing increased disk swelling). Consequently, if the ATPase itself is rate limiting, the rate of swelling should increase twofold. Figure 10 demonstrates that this is so. 


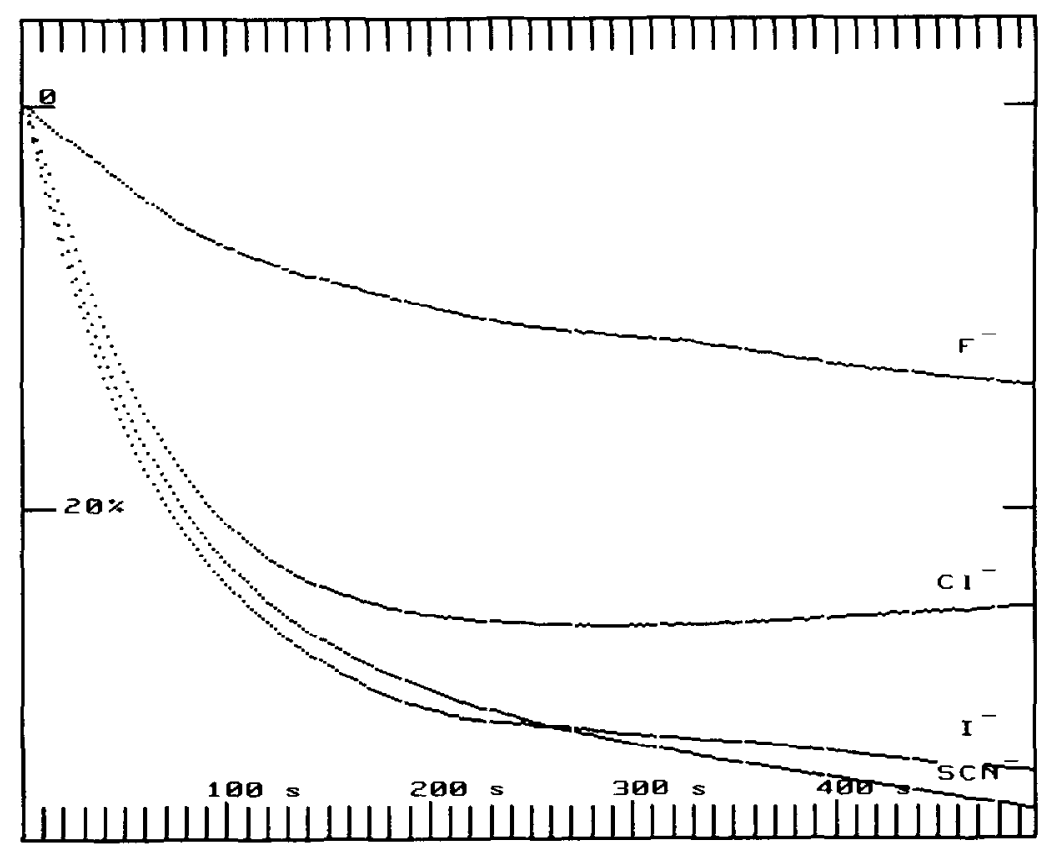

(a)

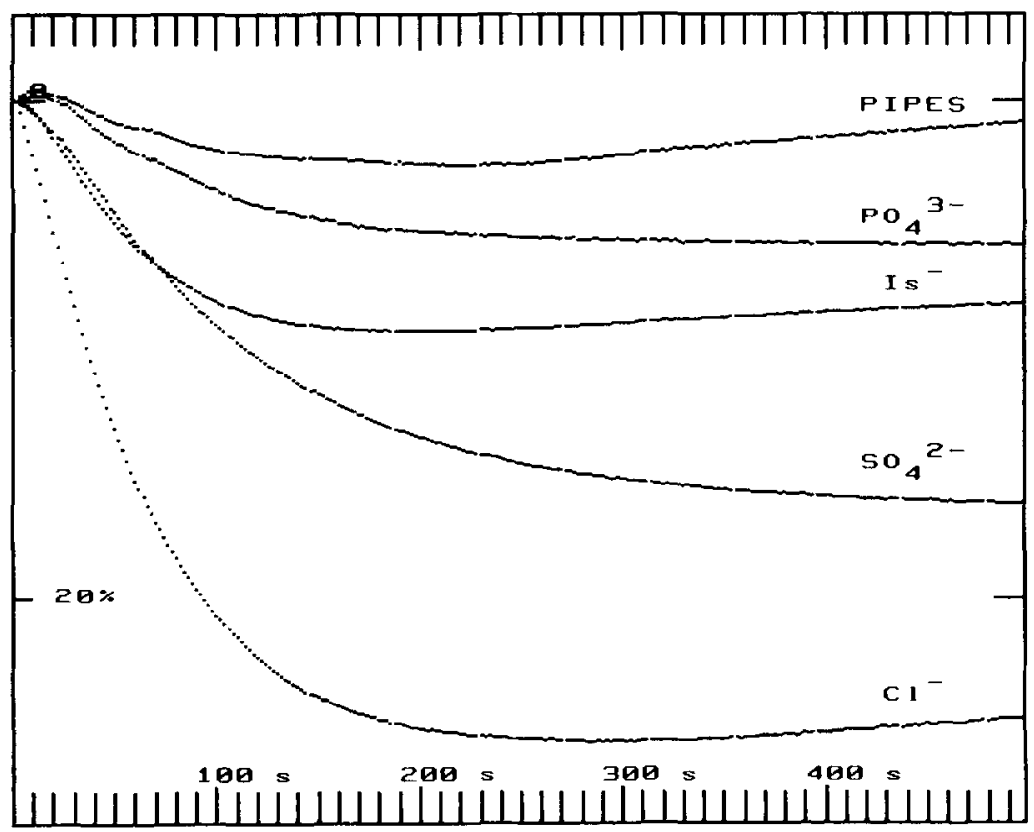

(b)

Fig. 9. $A_{\mathcal{D}}$ signals in the presence of various anions: (a) $90 \mathrm{mM}$ of the various potassium salts; (b) the potassium salt concentration of the various anions was chosen such that the total osmolarity was 200 mosM. The abbreviation Is $^{-}$denotes isethionate. 


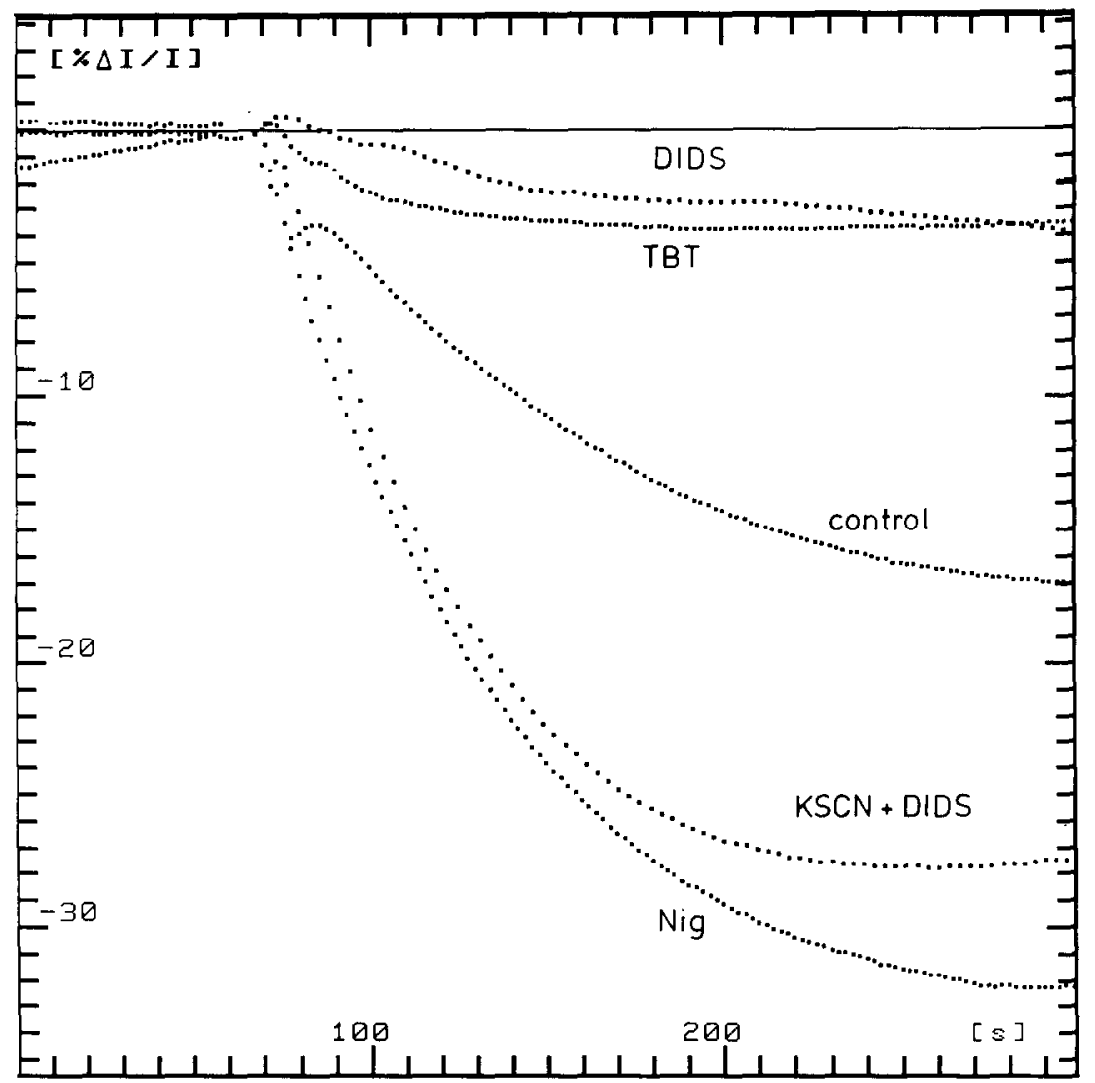

Fig. 10. $A_{D}$ signals in $100 \mathrm{mM} \mathrm{KCl}$, in the absence (control) and presence of $2 \mu \mathrm{M}$ nigericin (Nig), $1 \mu \mathrm{M}$ tributyltin (TBT), $50 \mu \mathrm{M}$ DIDS or $100 \mathrm{mM} \mathrm{KSCN}+50 \mu \mathrm{M}$ DIDS. ROS fragments were incubated for $5 \mathrm{~min}$ in the presence of the ionophores or the inhibitor before Mg-ATP was added.

\subsection{Dependence on $p H$}

Between $\mathrm{pH} 5.5$ and $8.0, \mathrm{~A}_{\mathrm{D}}$ is independent of the $\mathrm{pH}$ of the medium. Both above $\mathrm{pH} 8$ and below pH 5.5 its amplitude decreases; below $\mathrm{pH} 4.0$ its sign is reversed (Fig. 11).

\subsection{Dependence on light}

Qualitatively identical $A_{D}$ signals were obtained from unbleached and bleached ROS. The amplitude was usually slightly enhanced with bleached ROS (data not shown).

\subsection{Dependence on taurine}

There have been reports in the literature on an ATP-dependent, taurinestimulated calcium uptake by rod disks [25]. Therefore we tested the effect of taurine ( $50 \mathrm{mM}$ ) on $A_{D}$. No effect was found (data not shown). 


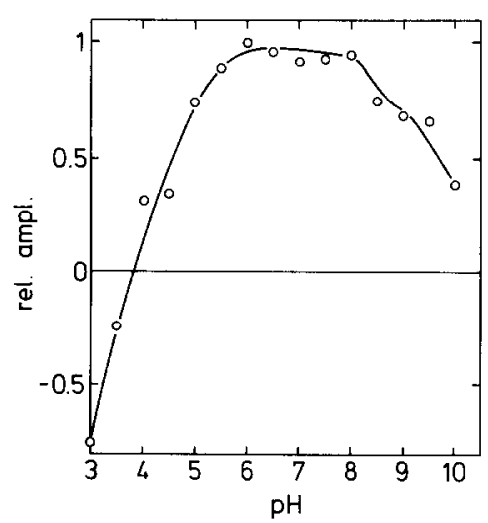

Fig. 11. Amplitude of as a function of $\mathrm{pH} \mathrm{A}_{\mathrm{D}}$ signal. The following buffers were used: pH $3.0-4.5 ; 5 \mathrm{mM}$ citric acid; $\mathrm{pH} 5.0-6.0 ; 5 \mathrm{mM}$ MES; $\mathrm{pH} 6.5-7.5 ; 5 \mathrm{mM}$ PIPES; pH $8.0-9.0 ; 5$ mM BICINE; pH $9.5-10.0 ; 5$ mM CAPS.

\section{Discussion}

A scattering signal as large as $A_{D}(20 \%-40 \%)$, which can be detected with the naked eye, clearly reflects a major structural reorganization. The experiments with freshly detached frog ROS indicate that in the living retina the energized state of the disk, i.e. its state after completion of the underlying processes of $A_{D}$, prevails and must therefore be considered to be physiological. This would seem to indicate that part of the huge metabolic energy supply in the photoreceptor cell is used for the setting up and maintenance of the proposed disk energization. Rod outer segments contain a nucleotide buffering system, and their usual nucleotide complement is $20 \mathrm{mM}$ creatine phosphate, $3 \mathrm{mM}$ ATP and $3 \mathrm{mM} \mathrm{GTP} \mathrm{[26].}$

There are two classes of transport ATPases in biological systems. The $\mathrm{F}_{1} / \mathrm{F}_{0}$-type ATPases of energy-conserving membranes, and the less complex transport ATPases found in other membranes. The latter are usually sensitive to vanadate, have no soluble protein subunits (like the $F_{1}$ complex) and form stable, phosphorylated intermediates [27]. Our ATPase still functions after hypotonic washing procedures and is inhibited by vanadate. It is probably identical with the vanadate-sensitive, phosphorylated, $160 \mathrm{kDa}$ protein described by Thacher [28]. It therefore belongs to the second category.

Most ATPases are transport ATPases, i.e. they promote transmembrane ion transport. The observation that $A_{D}$ signals can be obtained from freely floating, isolated disks indicates that the responsible ATPase is an integral part of the disk membrane and rules out a dynein-type ATPase located in connecting filaments [19]. This and the fact that typical transport ATPase inhibitors can block $A_{D}$ and the concomitant ATPase activity, points to a 
transport ATPase. Moreover, $A_{D}$ is affected by ionophores in an ion-and ionophore-specific way and is completely destroyed by reagents that break through the structural integrity of the disk compartment.

The question as to which ions are transported during the production of the $A_{D}$ signal can be answered, although not absolutely conclusively, on the basis of the light-scattering experiments. A vast amount of experimental evidence points to the proton as the primary transported ion: neither specific cations (apart from magnesium) nor specific anions are necessary for $A_{D}$ signals to occur. The DCCD sensitivity of the effect also confirms this, and the dramatic increase in signal amplitude in the presence of weak permeant bases such as imidazole suggests the same.

Proton transport across membranes can be electrogenic or electroneutral. The requirement for a permeant anion (either a lipid-soluble anion, such as thiocyanate, or chloride which obviously possesses specific carriermediated pathways in the disk) points to an electrogenic mechanism in the case of $A_{D}$.

When all the evidence is combined the following picture emerges. There is a vanadate- and DCCD-sensitive Mg-ATPase in the disk, which pumps protons into the disc interior. The proton uptake is electrically compensated by an anion uptake. Under physiological conditions this anion is chloride, which can cross the disk membrane through a specific DIDS-inhibited transport unit. As a result of the proton translocation a proton-motive force $\Delta \mu\left(\mathrm{H}^{+}\right)$develops across the disk membrane and also a chloride gradient. The $\mathrm{HCl}$ uptake leads to an osmotic swelling of the disks and it is this swelling which gives rise to the light-scattering signal $A_{D}$. Consequently, conditions which increase the (osmotically visible ) $\Delta \mathrm{pH}$ component of $\Delta \mu\left(\mathrm{H}^{+}\right)$(thiocyanate or iodide instead of chloride) enhance the amplitude of $A_{D}$. A similar enhancement is observed when valinomycin is administered in the presence of potassium: the swelling due to the uptake of $\mathrm{HCl}$ will dilute the internal potassium concentration and this will induce further swelling due to the uptake of $\mathrm{KCl}$. However, when valinomycin is administered in the presence of an impermeable cation, internal monovalent cations, which can be transported by valinomycin, are exchanged for the pumped protons and this, in turn, decreases the swelling and hence $A_{D}$.

When conditions are chosen such that $\mathrm{KCl}$ (in the presence of nigericin) or imidozolium chloride can be taken up instead of $\mathrm{HCl}$, the swelling is also enhanced due to the fact that only a small fraction of the internal protons are not bound and therefore osmotically active. In addition, under these conditions the ATPase can act longer and transport more ions before a $\Delta \mu(\mathrm{H} \downarrow)$ value has developed which is large enough to slow down the ATPase.

The rate-limiting step in the production of $A_{D}$ could either be the ATPase activity or the chloride transport. The fact that $A_{D}$ is not accelerated when chloride is replaced by thiocyanate points to the first possibility. Moreover, both $A_{D}$ signals and the concomitant ATPase activity start with a maximal rate. Therefore the electric field component of $\Delta \mu\left(\mathrm{H}^{+}\right)$should 
develop slowly during the production of $A_{D}$. This has been verified experimentally using field-sensitive dyes (data to be published).

Assuming a $1: 1$ or $2: 1$ stoichiometry between the number of pumped protons and the number of hydrolysed ATP molecules, there are approximately $4-8$ protons taken up per rhodopsin molecule during the production of $A_{D}$. This will be sufficient to protonate most or all of the available proton binding sites inside the disk [24].

The persistence of a small vanadate-sensitive (and DCCD-sensitive) ATPase activity after the completion of $A_{D}$ suggests the existence of a small leak in the disk membrane, most probably for protons. It corresponds to a flux of $280-560$ ions disk $\mathrm{s}^{-1} \mathrm{~s}^{-1}$. This low value is not in conflict with our previous observation of a strikingly low proton permeability of the disk [22]: even if the proposed $\Delta \mu\left(\mathrm{H}^{+}\right)$value amounts to only $10 \mathrm{mV}$, the leakage conductance of an entire disk will be in the range $4-8 \mathrm{fS}$ !

It is interesting to note that Bennett [29] has reached similar conclusions about $\Lambda_{\mathrm{D}}$, namely that ATP causes a chloride-dependent swelling of the disks. She has shown that this swelling is a prerequisite for a specific, light-induced, G-protein-related light-scattering signal. This signal, however, is distinct from the ATP-dependent light-scattering signal $A_{L}$, which probes the energized disk state with flashes of light, and which will be dealt with in detail in a following paper.

\section{Acknowledgment}

This work was supported by a research grant from the Deutsche Forschungs-Gemeinschaft (DFG, UH30/6,7) and a Heisenberg fellowship to R.U.

\section{References}

1 L. Stryer, Cyclic GMP cascade of vision, Annu. Rev. Neurosci., 9 (1986) 87 - 119.

2 M. L. Applebury and P. A. Hargrave, Molecular biology of the visual pigments, Vision Res., 26 (1986) 1881 - 1895.

3 K. P. Hofmann, R. Uhl, W. Hoffmann and W. Kreutz, Measurements of fast, lightinduced light-scattering and absorption changes in rod outer segments of vertebrate light sensitive rod cells, Biophys. Struct. Mech., 2 (1976) $61-77$.

4 R. Uhl, Lichtinduzierte Strukturänderungen in der Sehzelle, Ph.D. Thesis Universität Freiburg, 1976.

5 R. Uhl, K. P. Hofmann and W. Kreutz, Measurement of fast, light-induced discshrinkage within bovine rod outer segments by means of a light-scattering transient, Biochim. Biophys. Acta, 469 (1977) 113 - 122.

6 R. Uhl, K. P. Hofmann and W. Kreutz, On the light stimulated coupling between rhodopsin and its disk membrane environment, Biochemistry, 17 (1978) 5347 - 5352.

7 H. Kühn, N. Bennett, M. Michel-Villaz and M. Chambre, Interactions between photoexcited rhodopsin and GTP-binding protein: kinetic and stoichiometric analysis from light scattering changes, Proc. Natl. Acad. Sci. U.S.A., 78 (1981) 6873 - 6877. 
8 R. Uhl, T. Borys and E. W. Abrahamson, Rapid, light induced ATP-dependent processes in bovine rod outer segments, Biophys. J., 21 (1978) 126a.

9 R. Uhl, T. Borys and E. W. Abrahamson, Evidence for a Mg-dependent ATPase in bovine rod outer segment disk membranes, Photochem. Photobiol., 29 (1979) 703 706.

$10 \mathrm{R}$. Uhl, T. Borys and E. W. Abrahamson, Evidence for structural changes in the photoreceptor disk membrane, enabled by Mg-ATPase activity and triggered by light, FEBSLett., 107 (1979) 317 - 322.

11 R. Uhl, T. Borys and E. W. Abrahamson, Assays and characterisation of a Mg-ATPase in bovine rod outer segments, Methods Enzymol., 81 (1981) 509 - 513.

$12 \mathrm{R}$. Uhl, Über eine neue Mg-ATPase in Photorezeptor, Habilitation Thesis, Universität Göttingen, 1982.

13 R. Uhl, H. Desel and R. Wagner, Separation and characterisation of light scattering transients from rod outer segments of vertebrate photoreceptors, J. Biochem. Biophys. Methods, 11 (1.985) 1 - 13.

14 R. Uhl, H. Desel, N. Ryba and R. Wagner, A simple and rapid method for the preparation of intact bovine rod outer segments, J. Biochem. Biophys. Methods, 13 (1987) $127-138$.

15 H. G. Smith, G. W. Stubbs and B. J. Litman, The isolation and purification of osmotically intact discs from retinal rod outer segments, Exp. Eye Res., 20 (1975) $211-217$.

16 'I. Borys, Correlation of molecular and cellular events in visual photoreceptor cells, Ph.D. Thesis, University of Guelph, 1981.

17 R. E. Barnett, Effect of monovalent cations on the ouabain inhibition of the sodium and potassium ion activated, adenosine triphosphatase, Biochemestry, 9 (1970) $4644-4649$.

$18 \mathrm{~S}$. M. Thacher, Light stimulated, magnesium-dependent ATPase in toad rod outer segment, Biochemistry, 17 (1978) 3005 - 3009.

19 S. M. Thacher, ATP causes a structural change in retinal rod outer segment: disk swelling is not involved, J. Membr. Biol., 74 (1983) $95-102$.

20 D. Roof and J. E. Heuser, Surface of rod photoreceptor disk membrane: integral membrane components, J. Cell Biol., 95 (1982) 487 - 500.

21 K. P. Hofmann, A. Schleicher, D. Emeis and J. Reichert, Light-induced axial and radial shrinkage effects and changes of the refractive index in isolated bovine rod outer segments and dise vesicles, Biophys. Struct. Mech., 8 (1981) 67 - 93.

22 R. Uhl, P. V. Kuras, K. Anderson and E. W. Abrahamson, A light scattering study on the ion permeabilities of dark adapted bovine rod outer segments, Biochim. Biophys. Acta, 601 (1980) $462-477$.

23 A. L. Berman, A. M. Azimova and F. G. Gribakin, Localisation of $\mathrm{Na}^{+}-\mathrm{K}^{+}-\mathrm{ATPase}^{-}$ and $\mathrm{Ca}^{2+}$-activated Mg-ATPase in retinal rods, Vision Res., 17 (1977) $527-536$.

24 P. P. M. Schnetkamp, $\mathrm{Ca}^{2+}$ buffer sites in intact bovine rod outer segments: introduction to a novel optical probe to measure ionic permeabilities in suspensions of small particles, J. Membr. Biol., 88 (1985) 249 - 262.

25 C. H. Kuo and N. Miki, Stimulatory effect of taurine on calcium uptake by disk membranes from photoreceptor outer segments, Biochem. Biophys. Res. Commun., 94 (1979) $646-651$.

26 P. P. M. Schnetkamp and F. J. M. Daemen, Transfer of high energy phosphate in bovine rod outer segments, Biochim. Biophys. Acta, 672 (1981) $307-312$.

27 F. Schuurmans-Stekhoven and S. L. Bonting, Transport ATPases, Physiol. Rev., 61 (1981) $1-37$.

28 S. M. Thacher, Transient phosphorylation by ATP of a 160000 dalton protein in rod outer segments of the toad Bufo marinus, Biochim. Biophys. Acta, 648 (1981) $199-205$.

$29 \mathrm{~N}$. Bennett, A functional link between the dark Mg-ATPase activity and the light induced enzymatic cascade in rod outer segments, Eur. J. Biochem., 157 (1986) $487-495$. 